\title{
Industrial Internet of Things(IIoT)-Plattformtypen im Maschinen- und Anlagenbau
}

\author{
Laura Scheel (D) Benedict Bender
}

Eingegangen: 19. Mai 2021 / Angenommen: 11. November 2021 / Online publiziert: 7. Dezember 2021 (C) Der/die Autor(en) 2021

Zusammenfassung Das Angebot digitaler Plattformen ist mittlerweile auch im Maschinen- und Anlagenbau weit verbreitet. Dabei konnte in den letzten Jahren der Trend verzeichnet werden, dass die Herstellerunternehmen von Maschinen und Anlagen nicht mehr ausschließlich physische Produkte veräußern, sondern zusätzliche auf das Produkt abgestimmte Dienstleistungen, wie bspw. digitale Services. Dieser Wandel kann einen großen Einfluss auf die Veränderung des Geschäftsmodells haben und je nach Komplexität der digitalen Plattformen unterschiedliche Ausmaße annehmen, die auch strategische Entscheidungen bestimmen können. In diesem Beitrag wird eine Klassifizierung der digitalen Plattformen im deutschen Maschinenund Anlagenbau vorgenommen, mithilfe derer unterschiedliche Plattformtypen auf Grundlage ihrer Funktionszusammensetzung identifiziert werden. Demnach können bspw. Plattformen, über die lediglich grundlegende Funktionen wie die Verwaltung von Maschinen angeboten werden, von umfangreicheren Plattformen unterschieden werden, die eine höhere Komplexität aufweisen und somit einen größeren Einfluss auf die Veränderung des Geschäftsmodells haben. Diese Einteilung unterschiedlicher Plattformtypen kann Unternehmen im Maschinen- und Anlagenbau dabei unterstützen, strategische Entscheidungen bezüglich der Entwicklung und des Angebots digitaler Plattformen zu treffen und eine Einordnung ihrer digitalen Plattform im Wettbewerb vorzunehmen.

Schlüsselwörter Digitale Plattformen · Maschinen- und Anlagenbau • Industrie $4.0 \cdot$ IIoT $\cdot$ Plattformtypen

Laura Scheel $(\bowtie) \cdot$ Benedict Bender

Lehrstuhl für Wirtschaftsinformatik, insb. Prozesse und Systeme, Universität Potsdam,

August-Bebel-Str. 89, 14482 Potsdam, Deutschland

E-Mail: laura.scheel@wi.uni-potsdam.de

Benedict Bender

E-Mail: benedict.bender@wi.uni-potsdam.de 


\title{
Industrial Internet of Things (IIoT) Platform Types in Mechanical and Plant Engineering
}

\begin{abstract}
The offer of digital platforms has become very popular in mechanical and plant engineering. In recent years, there has been a trend for machinery and plant manufacturers to no longer sell only physical products, but also additional product-related services, such as digital services. This change can have a major impact on the transformation of the business model and can take on different dimensions depending on the complexity of the digital platforms, which can also determine strategic decisions. In this paper, a classification of digital platforms in the German machinery and plant engineering sector is made, with the help of which different platform types are identified based on their functions. Accordingly, platforms that only offer basic functions such as the management of machines can be distinguished from more extensive platforms that are more complex and thus have a greater influence on the change of the business model. This classification of different platform types can help companies in the mechanical and plant engineering sector to make strategic decisions regarding the development and offering of digital platforms and to classify their digital platform in the competitive environment.
\end{abstract}

Keywords Digital platforms · Machinery and plant engineering · Industry 4.0 • IIoT $\cdot$ Platform types

\section{Einleitung}

Das Industrial Internet of Things (IIoT) und das Angebot datenbasierter Vernetzung physischer Produkte haben die Geschäftsmodelle vieler Unternehmen verändert. Mithilfe des Internets der Dinge können diese Produkte bspw. auf Basis ihrer verarbeiteten Sensordaten kommunizieren und Prozesse dezentral gesteuert werden. Dabei können Innovationen wie digitale Plattformen verschiedene Bereiche eines Unternehmens grundlegend verändern (Oberländer et al. 2019; Pflaum und Schulz 2018).

Unter digitalen Plattformen werden im industriellen Kontext Produkt- und Serviceplattformen (häufig Cloud-basiert) verstanden, die Produktionskomponenten auf Grundlage von Datenaustausch miteinander vernetzen und dadurch digitale Services angeboten werden können (Oberländer et al. 2019; Obermaier und Mosch 2019). Fokus dieses Beitrags sind digitale Plattformen im deutschen Maschinen- und Anlagenbau. Das Angebot digitaler Plattformen im Maschinen- und Anlagenbau kann als Weiterentwicklung der Tertiarisierung angesehen werden, die in diesem Zusammenhang den seit einigen Jahren vorherrschenden Trend beschreibt, dass die Herstellerunternehmen nicht mehr lediglich ihre physischen Produkte veräußern, sondern zusätzlich auf das Produkt abgestimmte Dienstleistungen (z. B. Wartung und Reparaturen) anbieten, die nun auch vor allem datengetrieben sind (Bollhöfer et al. 2016). Dabei werden meistens bereits bestehende Produkte/Dienstleistungen durch digitale Elemente erweitert (Demont und Paulus-Rohmer 2017). Diese neuen Services können die Effizienz, Flexibilität und Agilität produzierender Unternehmen steigern 
(Pflaum und Schulz 2018). Mithilfe von digitalen Plattformen können Maschinenund Anlagenbauer ihren Kunden diese Services auf Grundlage der identifizierten Maschinendaten anbieten, was wiederum zu einer stärkeren Kundenbindung führt (VDMA et al. 2018).

Durch die rasante Ausbreitung dieser digitalen Innovationen steigt der Wettbewerb konkurrierender Unternehmen und die langfristige Weiterentwicklung der Geschäftsmodelle wird aufgrund des dadurch entstehenden Digitalisierungs- und Innovationsdrucks entscheidend für den Unternehmenserfolg (Oberländer et al. 2019). Demnach liegt eine wichtige strategische Entscheidung darin, die passend konzeptionierte digitale Plattform für das Unternehmen zu finden, die sowohl auf die Bedürfnisse des Anbieters und der Kunden als auch auf die Ressourcen abgestimmt ist.

Dabei unterscheiden sich digitale Plattformen im Maschinen- und Anlagenbau vor allem in den angebotenen Funktionen (Bollhöfer et al. 2016). Dadurch bilden die Funktionen bzw. die Funktionszusammensetzungen der Plattformen einen guten Anhaltspunkt für die Konzeption und Positionierung der eigenen digitalen Plattform, insbesondere im direkten Vergleich zu Wettbewerbern, die z. B. bei einer Marktanalyse identifiziert werden können. Bisher steht allerdings keine branchenspezifische Analyse der Plattformfunktionen zur Verfügung. Dementsprechend fehlt Unternehmen des Maschinen- und Anlagenbaus eine Grundlage für die zielgerichtete Zusammensetzung ihrer Plattform. Der vorliegende Beitrag greift diese Lücke auf und analysiert bereits angebotene Plattformen im Hinblick auf die Kombinationen ihrer Funktionen, um diese zu gruppieren und somit die Konzeption und Positionierung einer neuen digitalen Plattform zu erleichtern und zu unterstützen. Aus diesem Vorhaben ergibt sich die folgende Forschungsfrage:

Wie können digitale Plattform im deutschen Maschinen- und Anlagenbau auf Grundlage ihrer Funktionskombinationen gruppiert und unterschieden werden?

Die Veränderung und Erweiterung des Produktangebots mithilfe digitaler Komponenten können unterschiedliche Ausmaße annehmen (Oberländer et al. 2019). Dabei können Maschinen- und Anlagenbauer ihren Kunden mithilfe von digitalen Plattformen unterschiedlichste Funktionen bieten, die allerdings unterschiedlich komplex in der Entwicklung und der Umsetzung sein können. Während bspw. statusorientierte Funktionen, wie die Bereitstellung eines Dashboards, vornehmlich die einmalige Entwicklung von Software bedingen, so erfordert das Angebot von digitalen Wartungs- und Serviceleistungen neue unternehmensinterne Strukturen und die Vorhaltung entsprechender Kapazitäten. Dies zeigt, dass bestimmte Plattformfunktionen eine große Herausforderung bilden, vor allem für Unternehmen, für die das Angebot digitaler Produkte und Dienstleistungen eine Neuheit darstellt oder kleine und mittlere Unternehmen (KMU), z. B. in Bezug auf die Verfügbarkeit von notwendigen Ressourcen (Jun et al. 2017; Kammler et al. 2018). Dabei müssen nicht alle digitalen Plattformen komplexe Funktionen bereitstellen, sondern Unternehmen können sich auch auf grundlegende Funktionen berufen, die ihren Kunden bereits neue Mehrwerte bieten.

Dieser Beitrag und sein Ergebnis der Gruppierung digitaler Plattformen auf Grundlage ihrer Funktionskombinationen soll Maschinen- und Anlagenbauern einen vereinfachten Überblick über Möglichkeiten das Plattformangebots bieten. Dadurch 
kann die Komplexität von Marktanalysen der Wettbewerber reduziert oder Markteintrittsstrategien unterstützt werden, was insbesondere vor dem Hintergrund des sich schnell entwickelnden Digitalisierungsdrucks und dem damit einhergehenden Wettbewerb von großer Bedeutung ist.

Der Beitrag ist in fünf Kapitel strukturiert. Kapitel 2 stellt die typischen Funktionen digitaler Plattformen im Maschinen- und Anlagebau dar. Kapitel 3 erläutert die Methodik der Clusteranalyse. Kapitel 4 stellt die Analyse der identifizierten Cluster bzw. Typen digitaler Plattformen sowie deren Charakterisierung dar. Kapitel 5 stellt die Diskussion der Ergebnisse sowie des Beitrags für Forschung und Praxis dar. Ferner werden Limitationen und mögliche Ansätze für die zukünftige Forschung erläutert.

\section{Funktionen digitaler Plattformen}

Für das Forschungsvorhabens werden Plattformen im Bereich des deutschen Maschinen- und Anlagenbaus fokussiert. Im Kontext existierender Begriffsverständnisse kommt der Kontext der IIoT-Plattformen dem Untersuchungsbereich nahe. Der Fokus liegt auf Seite des Anbieters von Industriemaschinen, welcher Kunden eine digitale Plattform zur Anbindung seiner Maschinen bereitstellen möchte. Die Plattform soll zur Anbindung der Maschinen und darauf basierend für das Angebot von Zusatz- und Mehrwertdiensten genutzt werden.

Um das Ziel dieses Beitrags, die Kategorisierung von digitalen Plattformen im Maschinen- und Anlagenbau auf Grundlage ihrer Funktionen, zu verfolgen, wurde vorerst eine Literaturrecherche durchgeführt. Zum Beispiel führen Bollhöfer et al. (2016) verschiedene Geschäftsideen für die Industrie 4.0 im Maschinen- und Anlagenbau auf, in denen auch Plattformen eine zentrale Rolle spielen, z. B. als Kommunikationsschnittstelle, für den Zugriff auf Analysetools und anwendungsspezifisches Wissen oder als Marktplatz für Dienste und Technologiedaten. Lüttenberg et al. (2021) führen eine andere Kategorisierung digitaler Plattformstrategien an. Sie unterscheiden zwischen Smart-Data-Plattformen, die aggregierte und bereinigte Daten bereitstellen, Smart-Product-Plattformen, die den Zugriff auf maschinenspezifische Daten ermöglichen, sowie Matching-Plattformen, die als digitale Marktplätze verwendet werden, bei denen kein direkter Produktbezug besteht.

Hinter diesen Geschäftsideen stehen unterschiedliche Funktionen, die durch digitale Plattformen abgedeckt werden, bspw. sorgt eine Kommunikationsschnittstelle dafür, dass das Herstellerunternehmen der jeweiligen Maschine mit den Kunden im Fall eines Maschinenfehlers kommunizieren können. Diese Publikationen unterscheiden verschiedene Plattformkategorien, allerdings anhand einzelner Funktionen und nehmen dabei nicht in Betracht, dass über viele digitale Plattformen im Maschinen- und Anlagenbau verschiedenste Funktionen angeboten werden können, was eine Einordnung dieser Plattformen in die oben genannten Kategorien aus der Literatur erschwert.

Kammler et al. (2018) betrachten digitale Plattformen hingegen als Leistungsbündel, über das verschiedene Produkt- und Dienstleistungskomponenten angeboten werden können. Sie haben einen Anforderungskatalog an digitale Serviceplattformen 
im Maschinen- und Anlagenbau auf Basis einer Literaturrecherche und Experteninterviews im KMU-Umfeld erstellt. Unter diesen Anforderungen finden sich dabei konkrete Systemfunktionen, wie bspw. Fernwartungsfunktionen, eine Wissensdatenbank, Analysefunktionen, Predictive Maintenance, Kollaborationsfunktionen und die Integration bestehender Services, die u. a. Kernanforderungen an eine Serviceplattform darstellen (Kammler et al. 2018).

Dieser Anforderungskatalog stellte eine Grundlage für das Forschungsvorhaben dieses Beitrags und die Analyse der am Markt verfügbaren Plattformfunktionen dar. Auf Basis dessen war eine systematische Erhebung der digitalen Plattform im Markt erforderlich, um (1) die einzelnen Funktionen der angebotenen Plattformen zu identifizieren und zu definieren und (2) die Plattformen auf Grundlage dessen im Anschluss zu kategorisieren. Für diesen Beitrag wurde auf die bereits existierende Referenzdatenbank von Bender et al. (2021) zurückgegriffen, der eine systematische Marktrecherche durch ein Team mehrerer Wissenschaftler vorangegangen ist. Die Referenzdatenbank basiert auf der Identifikation von Referenzfällen durch Onlinerecherche, welche digitale Plattformen im deutschen Maschinen- und Anlagenbau darstellen. Diese Recherche beinhaltete das Branchenverzeichnis für den Maschinenund Anlagenbau des VDMA, branchenspezifische Statistiken, Fachmedien wie Zeitschriften und Messeverzeichnisse, und stichwortbasierte Onlinerecherche. Die Fälle der Referenzdatenbank wurden vorab qualifiziert, um insgesamt einheitliche Plattformen zu betrachten. Für die Qualifizierung wurden die fünf Kriterien: Hersteller, Konnektivität, Eigenständigkeit, Funktionalität und Multi-Device Fähigkeit genutzt. Für die identifizierten Plattformen und anbietenden Unternehmen wurden verschiedene relevante Merkmale und Parameter in die Referenzdatenbank aufgenommen, unter anderem die Subbranche des Unternehmens innerhalb des Maschinen- und Anlagenbaus, die Größe des Unternehmens, sowie die Bereitstellungsstrategie. Die Recherche resultierte in einer Grundgesamtheit von 116 digitalen Plattformen. Details über die Referenzdatenbank sowie die Methodik der Datenerhebung finden sich in Bender et al. (2021).

Um die Funktionen dieser digitalen Plattformen zu identifizieren, haben die $\mathrm{Au}$ toren dieses Beitrags die Referenzdatenbank erweitert und um die Funktionen der jeweiligen Plattform ergänzt, die auf Basis von öffentlich verfügbaren Informationen erhoben wurden, z. B. auf der Website des Anbieters oder in Berichterstattungen. Während der Recherche haben die Autoren sich in regelmäßigen Abständen ausgetauscht, um die Definitionen der identifizierten Funktionen aufeinander abzustimmen und zu vereinheitlichen. Dabei wurde schnell deutlich, dass sich die Funktionen und die dazugehörigen Definitionen vieler Plattformen ähneln, was die Recherche vereinfacht hat. Allerdings unterschieden sich die Plattformen sehr in der Anzahl sowie in der Komplexität der Funktionen, was die zuvor vermutete Heterogenität des Plattformangebots von deutschen Maschinen- und Anlagenbauern unterstützt.

Insgesamt konnten für 108 der recherchierten Fälle Funktionen identifiziert werden, welche die Grundgesamtheit für die folgende Analyse bilden. Im Zuge der Recherche konnten 11 Funktionen unterschieden und definiert werden, die bei den betrachteten Plattformen vielzählig vertreten und in Tab. 1 zusammengefasst sind. Die Erläuterungen der Funktionen basieren dabei auf der Zusammenfassung der 
Tab. 1 Funktionen digitaler Plattformen im Maschinen- und Anlagenbau

\begin{tabular}{|c|c|}
\hline Funktion & Erläuterung \\
\hline $\begin{array}{l}\text { Verwaltung von Res- } \\
\text { sourcen und Maschinen }\end{array}$ & $\begin{array}{l}\text { Mithilfe der digitalen Plattform können Status- und Komponenteninforma- } \\
\text { tionen verwaltet werden und/oder der Maschinenpark und Maschinenstand- } \\
\text { ort angezeigt werden }\end{array}$ \\
\hline Event-Management & $\begin{array}{l}\text { Die digitale Plattform benachrichtigt oder alarmiert die Nutzer bei einem } \\
\text { Maschinenfehler, wie bspw. einer Schwellwertüberschreitung }\end{array}$ \\
\hline $\begin{array}{l}\text { Analyse und Zustands- } \\
\text { überwachung }\end{array}$ & $\begin{array}{l}\text { Die Maschinen und/oder Produktionsumgebungen können anhand bestimm- } \\
\text { ter Parameter analysiert werden und/oder Berichte erzeugt werden }\end{array}$ \\
\hline $\begin{array}{l}\text { Predictive Monitoring/ } \\
\text { Maintenance }\end{array}$ & $\begin{array}{l}\text { Die Maschinendaten werden genutzt, um eine automatisierte vorausschauen- } \\
\text { de Wartung zu planen }\end{array}$ \\
\hline Visualisierung & $\begin{array}{l}\text { Die aufbereiteten Daten, z. B. Key Performance Indicators, können mithilfe } \\
\text { von Dashboards visuell dargestellt werden }\end{array}$ \\
\hline Wissensdatenbank & $\begin{array}{l}\text { In die digitale Plattform ist eine Wissensdatenbank integriert, um bspw. } \\
\text { einen Zugriff auf Anleitungen, erklärende Videos oder Best Practices zu } \\
\text { gewährleisten }\end{array}$ \\
\hline $\begin{array}{l}\text { Steuerung von Maschi- } \\
\text { nen }\end{array}$ & $\begin{array}{l}\text { Es ist ein aktiver Zugriff auf bestimmte Maschinen und Anlagen über die } \\
\text { digitale Plattform möglich, um zur Steuerung in den Betrieb einzugreifen }\end{array}$ \\
\hline $\begin{array}{l}\text { Kollaboration zwischen } \\
\text { Kunden und Maschi- } \\
\text { nenhersteller }\end{array}$ & $\begin{array}{l}\text { Die Kunden können, z. B. im Fall einer defekten Maschine oder eines Aus- } \\
\text { falls, über die digitale Plattform mit dem Hersteller kommunizieren und/oder } \\
\text { es werden automatische Tickets bei dem Hersteller erzeugt }\end{array}$ \\
\hline $\begin{array}{l}\text { Wartung und andere } \\
\text { Services }\end{array}$ & $\begin{array}{l}\text { Wartungen werden dokumentiert und/oder es können remote Services über } \\
\text { die digitale Plattform angeboten werden }\end{array}$ \\
\hline Zugang zu einem Shop & $\begin{array}{l}\text { Ein direkter Zugang zu einem Shop, um bspw. Ersatzteile bei einer defekten } \\
\text { Maschine direkt bestellen zu können }\end{array}$ \\
\hline Integrationsmöglichkeiten & $\begin{array}{l}\text { Eine Integration mit anderen Systemen, bspw. über Schnittstellen oder } \\
\text { Webservices, ist möglich }\end{array}$ \\
\hline
\end{tabular}

Definitionen verschiedener Plattformen, die durch die Internetrecherche entwickelt wurden.

\section{Methodik}

Die in Tab. 1 dargestellten Plattformfunktionen und die digitalen Plattformen der vorgestellten Referenzdatenbank bilden die Basis für die nun folgende Kategorisierung der Plattformen anhand ihrer Funktionskombinationen.

\subsection{Datenaufbereitung}

Nachdem die Funktionen der verschiedenen Plattformen ermittelt wurden, sollte im nächsten Schritt eine Clusteranalyse durchgeführt werden. Die Methodik der Clusteranalyse folgt dabei den Vorgaben von Backhaus et al. (2018). Für die Analyse ist es von Bedeutung, dass die definierten Plattformfunktionen als Objekte keine gegenseitigen Abhängigkeiten aufweisen. Sowohl die Visualisierung von Maschinendaten als auch die vorausschauende Wartung sind von einer vorangestellten Analyse der Maschinendaten abhängig, somit besteht eine Abhängigkeit von der Funktion Analyse und Zustandsüberwachung. Aus diesem Grund wurden die Funk- 
tionen Visualisierung und Predictive Monitoring/Maintenance aus der folgenden Analyse ausgeschlossen und aus dem Datensatz entfernt. Alle anderen Funktionen weisen keine Abhängigkeiten von anderen Funktionen auf und können somit bei der Analyse berücksichtigt werden.

Die Daten liegen in Form einer binären Variablenstruktur vor, d.h. alle Merkmalsvariablen nehmen die Ausprägung Null oder Eins an. Dabei bedeutet der Wert Eins „die Plattform beinhaltet diese Funktion“ und Null „die Plattform beinhaltet diese Funktion nicht“. Mit dieser Struktur sind die Daten für die folgende Clusteranalyse manuell aufbereitet worden.

\subsection{Clusteranalyse}

Um verschiedene Typen der digitalen Plattformen im Maschinen- und Anlagenbau zu identifizieren, wurde die Clusteranalyse als exploratives multivariates Analyseverfahren gewählt. Dabei bildet die Grundlage eine Matrix aus den 108 Plattformen und den neun definierten Plattformfunktionen mit den binären Variablen Null und Eins. Die Clusteranalyse wurde in der Statistikumgebung R umgesetzt. In der vorliegenden Studie wurde die K-Means Clusteranalyse als partitionierendes Verfahren gewählt, da sie die vorherige Festlegung der Clusteranzahl als Anfangspartition ermöglicht, und die Clusterzugehörigkeit im Fusionierungsprozess verbessert wird.

Die Anfangspartition wurde optisch mithilfe des Elbow-Kriteriums bestimmt (Scree-Plot). In einem Koordinatensystem wurde dabei die Fehlerquadratsumme gegen die zugehörige Clusterzahl abgetragen (Abb. 1). Nach dem Elbow-Kriterium ist eine Fünf-Cluster-Lösung zu wählen.

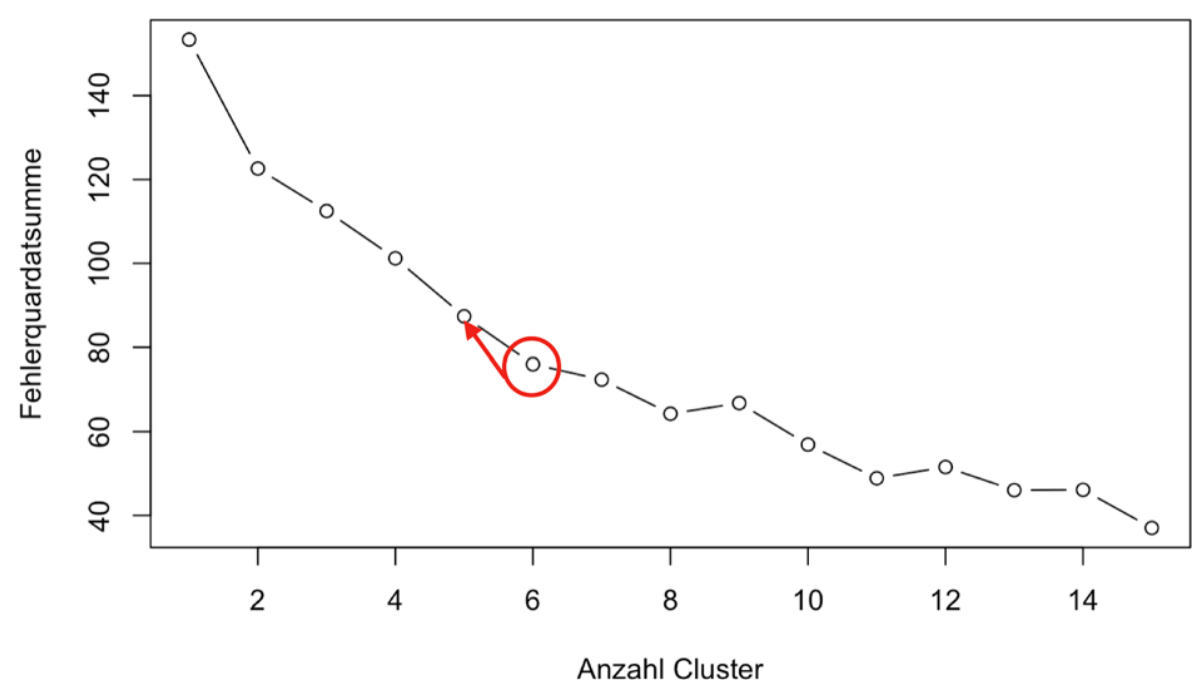

Abb. 1 Scree-Plot zur Festlegung der Anzahl der Cluster 


\section{Analyseergebnisse}

\subsection{Deskriptive Statistik}

Hinsichtlich der Unternehmensgröße sind 78\% Großunternehmen und der Rest KMU (24). Abb. 2 stellt die Verteilung der Referenzfälle bezüglich der Subbranche im Maschinen- und Anlagenbau dar.

Domäne

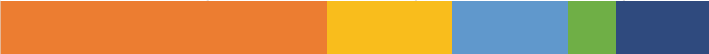

$0 \%$

$20 \%$

$40 \%$

$60 \%$

$80 \%$

$100 \%$

- Werkzeugmaschinen

- Mischkonzern

- Antriebstechnik
Bergbau-, Bau- und Baustoffmaschinen

- Landmaschinen

Sonstige

Abb. 2 Charakterisierung der Referenzfälle nach Spezialisierung

\begin{tabular}{|c|c|c|c|c|c|}
\hline Funktion & Cluster 1a (1) & Cluster 1b (2) & Cluster 2 (3) & Cluster 3 (4) & Cluster 4 (5) \\
\hline $\begin{array}{l}\text { Verwaltung von } \\
\text { Ressourcen und } \\
\text { Maschinen }\end{array}$ & 1,00 & 0,83 & 0,95 & 1,00 & 1,00 \\
\hline $\begin{array}{c}\text { Event- } \\
\text { Management }\end{array}$ & 1,00 & 0,00 & 0,00 & 0,93 & 0,58 \\
\hline $\begin{array}{l}\text { Analyse / } \\
\text { Zustands- } \\
\text { überwachung }\end{array}$ & 1,00 & 0,94 & 1,00 & 1,00 & 0,95 \\
\hline Wissensdatenbank & 0,00 & 0,06 & 0,09 & 0,43 & 0,05 \\
\hline $\begin{array}{l}\text { Steuerungs- } \\
\text { funktion }\end{array}$ & 0,37 & 0,06 & 0,41 & 0,07 & 0,32 \\
\hline $\begin{array}{c}\text { Kollaboration } \\
\text { zwischen Kunde } \\
\text { und Hersteller }\end{array}$ & 0,00 & 0,06 & 0,00 & 0,57 & 1,00 \\
\hline $\begin{array}{l}\text { Wartung / } \\
\text { Services }\end{array}$ & 0,60 & 0,39 & 0,50 & 1,00 & 1,00 \\
\hline Zugang zum Shop & 0,03 & 0,11 & 0,09 & 1,00 & 0,11 \\
\hline $\begin{array}{l}\text { Integrations- } \\
\text { möglichkeiten mit } \\
\text { anderen Systemen }\end{array}$ & 0,54 & 0,00 & 1,00 & 0,29 & 0,58 \\
\hline
\end{tabular}

Abb. 3 Verteilung der Plattformfunktionen je Cluster 
Tab. 2 Allgemeine Clusterdaten

\begin{tabular}{llllll}
\hline Ursprüngliche Nummerierung der Cluster & Cluster 1 & Cluster 2 & Cluster 3 & Cluster 4 & Cluster 5 \\
Neue Nummerierung der Cluster & Cluster 1a & Cluster 1b & Cluster 2 & Cluster 3 & Cluster 4 \\
$\begin{array}{l}\text { Anzahl der Plattformen } \\
\begin{array}{l}\text { Durchschnittliche Anzahl an Funktionen } \\
\text { der Plattformen }\end{array}\end{array}$ & 45 & 18 & 22 & 14 & 19 \\
\hline
\end{tabular}

Die Verteilung der Plattformfunktionen in den fünf identifizierten Clustern als Ergebnis der statistischen Clusteranalyse wird in Abb. 3 dargestellt. Die abgebildeten Zahlen symbolisieren dabei den durchschnittlichen Wert der binären Variable Eins „die Plattformen beinhalten diese Funktion“ und Null „die Plattformen beinhalten diese Funktion nicht" innerhalb des jeweiligen Clusters. Zur visuellen Veranschaulichung wurden die Werte durch Farben unterstützt: Je dunkler die Farbe ist, desto stärker ist die Funktion in dem Cluster vertreten und kann das jeweilige Cluster charakterisieren.

Da die Unterschiede in der Funktionskombination zwischen den anderen Clustern größer sind, wurden die Cluster 1 und 2 zu den Subclustern 1a und $1 \mathrm{~b}$ umbenannt (siehe Tab. 2). Diese neue Nummerierung findet von nun an im Verlauf des Beitrags Anwendung. Abb. 3 verdeutlicht, dass die Cluster 1a und 1b hauptsächlich Funktionen beinhalten, die als grundlegend bezeichnet werden können, da sie in allen Clustern stark vertreten sind. Dabei sind insbesondere die Verwaltung von Ressourcen und Maschinen sowie die Analyse und Zustandsüberwachung zu betrachten.

Die Cluster 2, 3 und 4 grenzen sich dem entgegen mit einer höheren Anzahl zusätzlicher Funktionen ab, die als spezieller bezeichnet werden können, wie bspw. die Integrationsmöglichkeiten mit anderen Systemen in Cluster 2, Wartung/Services und ein direkter Shop-Zugang in Cluster 3 sowie die Möglichkeit der Kollaboration zwischen Kunde und Hersteller und Wartung/Services in Cluster 4.

Neben den grundlegenden Funktionen bildet Abb. 3 auch andere Funktionskombinationen $a b$, die häufig gemeinsam angeboten werden. So ist bspw. ersichtlich, dass die Steuerungsfunktion über die digitale Plattform angeboten wird, wenn auch Integrationsmöglichkeiten mit anderen Systemen bestehen. Dabei ist denkbar, dass die Steuerung über andere Systeme stattfindet.

Tab. 2 verdeutlicht weiterhin, dass die einzelnen Cluster geringe Unterschiede in ihrer Größe aufweisen. Zudem kann festgestellt werden, dass die Anzahl der Funktionen der Plattformen zwischen den Clustern variiert. Demnach haben die Plattformen innerhalb des Clusters 1b die geringste Anzahl an Funktionen, während die Plattformen in Cluster 3 die höchste Anzahl an Funktionen aufweisen können.

\subsection{Klassifizierung der Plattformen}

Anhand der Funktionen bzw. Funktionskombinationen und der beschriebenen Einflussfaktoren sollen die verschiedenen Plattformtypen nun näher beschrieben und charakterisiert werden. Als zusätzliche Charakterisierungsmerkmale der Cluster werden dabei zudem die Unternehmensgröße, die Bereitstellungsstrategie der Plattform, sowie die Subbranche des anbietenden Unternehmens innerhalb des Maschinen- und Anlagenbaus betrachtet. Dabei werden drei Bereitstellungsstrategien unterschieden: 


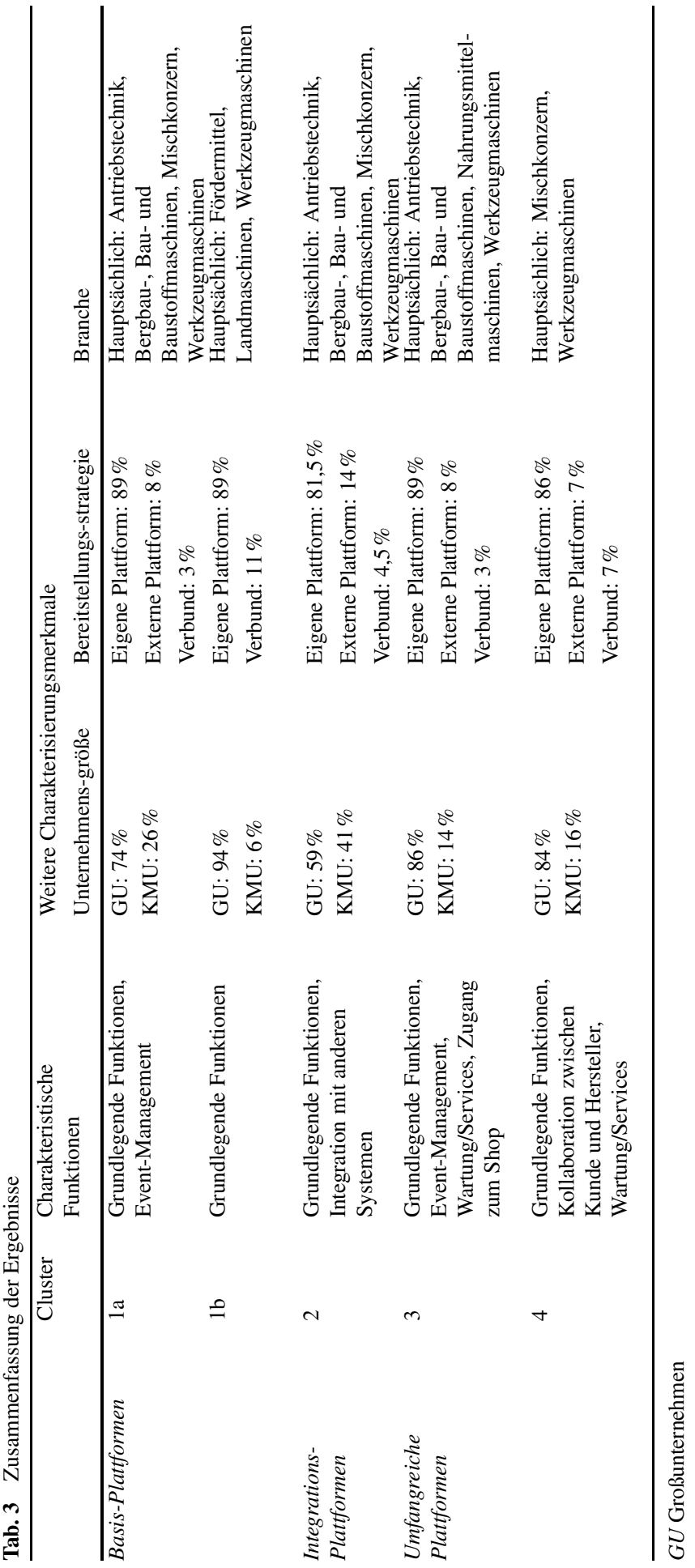


Das Unternehmen bietet eine komplett selbstentwickelte Plattform an, das Unternehmen bietet eine externe, fremdentwickelte Plattform an, oder es wird eine Plattform im Verbund gemeinsam mit anderen Unternehmen angeboten. Außerdem wird in diesem Beitrag zwischen 15 Subbranchen des Maschinen- und Anlagenbaus, die sich hauptsächlich an der Einteilung des Statistischen Bundesamts (2021) orientieren, und sonstigen Unternehmen, bei der keine Zuordnung zu einer Subbranche möglich war, unterschieden. Die Subbranchen sowie ihre Clusterverteilung sind in der Tabelle im Anhang dargestellt. Bei dieser Darstellung muss beachtet werden, dass das Cluster 1a mit 35 Plattformen das mit Abstand größte Cluster ist und die Betrachtung der verteilten Clusteranteile über die Subbranchen verzerrt werden könnte. Tab. 3 bietet einen Überblick über die nun vorgestellten Ergebnisse.

\subsubsection{Cluster 1a und $1 b$}

Die Cluster 1a und 1b decken hauptsächlich grundlegende Funktionen ab, wie die Verwaltung von Ressourcen und Maschinen sowie die Analyse und Zustandsüberwachung. Cluster 1a weist zudem einen hohen Anteil der Funktion Event-Management auf.

Mit 35 Plattformen ist das Cluster 1a das mit Abstand größte Cluster und zeichnet sich durch keine besonderen Auffälligkeiten bzgl. der Unternehmensgröße, der Plattform-Bereitstellungsstrategie oder der Subbranche der anbietenden Unternehmen aus. Aus diesem Grund kann angenommen werden, dass sich in diesem Cluster hauptsächlich Plattformen befinden, die nicht eindeutig einem der anderen Cluster zugeordnet werden können.

Cluster 1b beinhaltet fast nur Großunternehmen (94\%) und ist das einzige Cluster, das keine externen Plattformen beinhaltet. Der Mangel an externen Plattformen und der im Gegensatz dazu hohe Anteil an Eigenentwicklungen ist dabei kompatibel mit den Subbranchen des Maschinen- und Anlagenbaus, die sich in diesem Cluster befinden. Demnach können die Maschinen mit einem hohen Anteil an Landmaschinen und Fördermitteln als spezialisiert bezeichnet werden. Zudem beinhaltet dieses Cluster keine Plattformen von Mischkonzernen, die u. U. weniger spezialisiert sind und somit extern bezogen werden können.

Die beiden Cluster 1a und 1b grenzen sich von den anderen Clustern vor allem durch die grundlegenden Funktionen ihrer Plattformen ab. Zudem befinden sich insbesondere in Cluster $1 \mathrm{~b}$ im Vergleich zu den anderen Clustern Plattformen mit deutlich weniger Funktionen $(\varnothing 2,44)$. Somit können mit diesen digitalen Plattformen lediglich grundlegende Funktionen abgedeckt werden und nur in einem geringen Umfang die digitalen Services, die zu einer größeren Veränderung des Geschäftsmodells führen würden.

$\mathrm{Zu}$ Beispielen aus der vorgestellten Referenzdatenbank, die den Clustern 1a oder 1b zugeordnet werden, gehören die KMU Certuss Dampfautomaten GmbH \& Co. KG (Telekom Deutschland GmbH 2021) und Petko GmbH (Weidmann 2019), die jeweils beide die Cloud der Dinge der Telekom anbieten, oder auch das Großunternehmen KUKA AG mit seiner Plattform KUKA Connect (KUKA AG 2021). 


\subsubsection{Cluster 2}

Neben grundlegenden Funktionen befindet sich in Cluster 2 die höchste Dichte an Plattformen mit Steuerungsfunktionen und Integrationsmöglichkeiten mit anderen Systemen. Insbesondere die Möglichkeit, die Plattformen in andere Systeme zu integrieren passt auch zu der Größe der Unternehmen, deren Plattformen in diesem Cluster vertreten sind. So befindet sich im Cluster 2 ein verhältnismäßig großer Anteil an KMU mit $41 \%$. Bei KMU ist davon auszugehen, dass die digitalen Plattformen eher als kleinere Software angeboten werden, die als Integrationskomponente zu anderen Systemen fungiert als eine eigenständige Komplettlösung. Zudem besteht in diesem Cluster ein vergleichsweise hoher Anteil an externen Plattformen (14\%) und der geringste Anteil eigener Plattformen (81,5\%), was auch damit zusammenhängen könnte, dass KMU häufig die notwendigen personellen und finanziellen Ressourcen fehlen, um die digitalen Plattformen selbst zu entwickeln.

Das Plattformtyp des Clusters 2 kann somit als spezialisierter als Cluster 1a und $1 \mathrm{~b}$ angesehen werden, wobei den Kunden neben den grundlegenden Funktionen weitere Mehrwerte angeboten werden, wie bspw. die Anlagen und Maschinen über die digitale Plattform steuern zu können.

Als Referenzplattformen für Cluster 2 können die digitalen Plattformen VEN Profit von Venjakob Maschinenbau GmbH \& Co. KG (Venjakob Maschinenbau $\mathrm{GmbH} \&$ Co. KG 2021) und Big-LinX von ads-tec Industrial IT GmbH (ads-tec Industrial IT GmbH 2021) angeführt werden. Beide Unternehmen sind KMU.

\subsubsection{Cluster 3}

Cluster 3 beinhaltet mit durchschnittlich 6,29 Funktionen die Plattformen mit der höchsten Funktionsvielfalt. In diesem Cluster befinden sich, neben den grundlegenden Funktionen, auch eine hohe Anzahl an Plattformen mit der Funktion für Wartung/Services, sowie die verhältnismäßig höchste Anzahl an den Funktionen Wissensdatenbank und direkter Zugang zu einem Shop. Auch die Subbranchen des Maschinen- und Anlagenbaus, die diese Plattformen anbieten, sind sehr divers und weisen keine besonderen Auffälligkeiten auf.

Mit seinem diversen Funktionsangebot bietet dieser Plattformtyp im Verhältnis zu den anderen den größten Mehrwert für die Kunden, da diese Plattformen eine Vielzahl an zusätzlichen Services bieten. Dies bedeutet wiederum auch, dass die Maschinenhersteller, die diese Plattformen anbieten, ihr Geschäftsmodell und ihre Prozesse an diese zusätzlichen digitalen Services anpassen müssen.

Zum Beispiel können die digitalen Plattformen Heidelberg Assistant (Heidelberger Druckmaschinen AG 2021) und SERICY (Grenzebach Digital GmbH 2021) der Großunternehmen Heidelberger Druckmaschinen AG und Grenzebach Digital $\mathrm{GmbH}$ als Referenzen für dieses Cluster angeführt werden.

\subsubsection{Cluster 4}

Auch mit dem Plattformtyp in Cluster 4 können zusätzliche Services angeboten werden. Über diesen digitalen Plattformtyp können neben grundlegenden Funktionen 
auch Wartungen und ähnliche Services durchgeführt werden. Dieses Cluster zeichnet zudem aus, dass es den höchsten Anteil der Kollaborations-Funktion beinhaltet, mit der die Kunden mit dem Herstellerunternehmen über die Plattform kommunizieren können. Mit $42 \%$ befindet sich in Cluster 4 der höchste Anteil an Mischkonzernen im Vergleich zu anderen Clustern, was zeigt, dass dieser Plattformtyp bei verschiedenen Maschinenarten Anwendung finden kann.

Die digitalen Plattformen LOOXR Druckluft 4.0 des KMU Mader GmbH \& Co. KG (Mader GmbH \& Co. KG 2021) und rConnect des Großunternehmens GF Machining Solutions GmbH (GF Machining Solutions Management SA 2021) sind Beispiele für Plattformen des Clusters 4.

Durch die vorgenommene Analyse konnten verschiedene Plattformtypen identifiziert werden, die sich vor allem durch ihre unterschiedlichen Funktionen auszeichnen. Tab. 3 fasst die verschiedenen Cluster zusammen.

\section{Diskussion}

Die Analyse und Interpretation der Cluster, bei der die Funktionen sowie verschiedene Einflussfaktoren der anbietenden Unternehmen näher betrachtet wurden, lässt eine gröbere Einteilung der Cluster in drei Gruppen zu. Demnach können die beiden Cluster 1a und 1b, wie bereits beschrieben, zu der Gruppe Basis-Plattformen zusammengefasst werden, da sie im Gegensatz zu den anderen Clustern nur grundlegende Funktionen abdecken. Des Weiteren unterscheidet sich Cluster 2 von den anderen Clustern und bildet somit als einziges Cluster die Gruppe der IntegrationsPlattformen, was durch die charakteristische Funktion dieses Clusters beeinflusst ist. Die letzten beiden Cluster 3 und 4 können zu der Gruppe Umfangreiche Plattformen zusammengefasst werden, da sie die größte Vielfalt an Plattformfunktionen aufweisen und den Kunden somit den größten Mehrwert bieten können. Mithilfe dieser Plattformen werden die Möglichkeiten des IIoT und des Angebots digitaler Services zum aktuellen Zeitpunkt im deutschsprachigen Maschinen- und Anlagenbau größtmöglich ausgenutzt.

Die Dreiteilung der Plattformtypen in Basis-Plattformen, Integrations-Plattformen und umfangreiche Plattformen (siehe Tab. 3) in dieser Reihenfolge weist eine steigende Komplexität auf. D.h., dass nicht nur die Anzahl der Funktionen, die durch diese Plattformen angeboten werden, steigt, sondern vor allem die Komplexität dieser. Für die anbietenden Unternehmen bedeutet dies, dass bspw. für das Angebot einer umfangreichen Plattform deutlich mehr innerbetriebliche Strukturen und Prozesse verändert sowie mehr Ressourcen aufgewendet werden müssen als für das Angebot einer Basis-Plattform. Dabei besteht die Veränderung nicht nur darin, dass ein neues technisches Produkt angeboten wird, sondern es entstehen bspw. neue Partnerschaften zu anderen Unternehmen, erweiterte Kundenbindungen, sowie neue Preis- und Ertragsmodelle.

Entscheidet sich ein Unternehmen aus dem Maschinen- und Anlagenbau dafür, eine Basis-Plattform anzubieten, ist der Einfluss auf das Geschäftsmodell, die Strukturen und Prozesse gering. Hierbei besteht die Veränderung hauptsächlich in der Bereitstellung eines neuen technischen Produkts und nicht in dem Angebot neu- 
er Services, da das herstellende Unternehmen nicht in die Nutzung der Plattform involviert ist, wie bspw. bei der Funktion Wartung/Services, bei der der Maschinenhersteller zusätzliche Services zur Verfügung stellen muss. Trotzdem müssen für das Angebot dieses Plattformtyps finanzielle und personelle Ressourcen verfügbar sein. Bei der Bereitstellungsstrategie der externen Plattform müssen zudem neue Partnerschaften mit anderen Unternehmen eingegangen werden, um die Plattform bereitzustellen. Außerdem muss ein Preismodell entwickelt werden, um mit der digitalen Plattform bestimmte Erträge zu erzielen. Dabei haben Modelle wie bspw. Pay-per-use, Freemium oder Einmalkauf unterschiedliche Auswirkungen auf den Ertrag.

Auch das Angebot einer Integrations-Plattform beeinträchtigt die Veränderungen nur in geringem Maße. Hierbei muss jedoch beachtet werden, dass insbesondere KMU, die in diesem Cluster im Gegensatz zu den anderen Clustern mehr vertreten sind, häufig nicht über die Ressourcen verfügen, um die Idee einer digitalen Plattform zu realisieren. Demnach befinden sich in größeren Unternehmen häufig bereits vor dem Plattformangebot IT- oder Forschungsabteilungen, die sich mit Digitalisierungsthemen oder neuen Innovationen befassen. Dabei müssen KMU in manchen Fällen auf externes Wissen oder Beratungen zurückgreifen. Für KMU besteht der wesentliche Vorteil des Angebots einer Integrations-Plattform besonders darin, dass sie hauptsächlich sehr spezialisierte Maschinen anbieten, die ihre Kunden in einem komplexen Gesamtprozess integrieren müssen. Hierbei könnte eine Integrations-Plattform den Automatisierungsaufwand ggf. reduzieren, da die Integration der digitalen Plattform als Teil des Gesamtportfolios an Maschinen die Komplexität vermindert.

Den größten Einfluss auf das Geschäftsmodell verzeichnen die umfangreichen Plattformen. Während bei den anderen Plattformtypen hauptsächlich grundlegende Funktionen angeboten werden, bieten diese Plattformen weitere Services und Funktionen, die zu weiteren Prozessveränderungen innerhalb der herstellenden Unternehmen führen können. Zum Beispiel müssen für die Kollaborations-Funktion neue Prozesse, wie bspw. ein Live-Chat und dementsprechendes Personal, implementiert werden, um diesen Service anzubieten. Ähnlich ist dies auch auf das Angebot von Remote-Wartungen anwendbar. So müssen hierfür u. U. neue personelle Ressourcen zur Verfügung stehen, die für die Technik geschult werden müssen. Auch für einen direkten Zugang zu einem Shop für Ersatzteile müssen Systeme umgesetzt werden, die diese automatischen Bestellungen abwickeln und bearbeiten. Zudem hat das Angebot zusätzlicher digitaler Services eine Auswirkung auf die Kundenbindung und den näheren Kontakt mit den Kunden, insbesondere dann, wenn die Unternehmen vor dem Einsatz der digitalen Plattformen noch nicht ähnliche Services angeboten, sondern lediglich die Maschinen ohne produktnahe Services verkauft haben.

Die unterschiedlichen Abstufungen der Komplexität der Plattformtypen machen deutlich, dass die unterschiedlichen Cluster auch eine Weiterentwicklung darstellen könnten oder sich diese Abstufung als Bestandteil einer möglichen Markteintrittsstrategie eignet. Vor allem für Unternehmen, die vor der Entscheidung stehen, eine digitale Plattform anzubieten und bisher nur wenig Erfahrungen mit dem Angebot digitaler Produkte gesammelt haben, stellen die in diesem Beitrag identifizierten Plattformtypen bzw. Kategorien einen Anhaltspunkt dar, um die eigene Plattform 
zu konzeptionieren und im Wettbewerb zu positionieren. So stellen die einzelnen Cluster zusätzlich eine grobe Marktübersicht dar, die Unternehmen im Maschinenund Anlagenbau dabei unterstützen kann, sich in ihren Wettbewerb, z. B. auf Grundlage ihrer Branche oder Unternehmensgröße, einzuordnen. Aufgrund der steigenden Konkurrenz um digitale und innovative Angebote im Maschinen- und Anlagenbau stellt dies sogar eine notwendige Maßnahme dar, um den langfristigen Unternehmenserfolg sicherzustellen.

Zum Beispiel ist das Angebot einer umfangreichen Plattform nach dieser Analyse für KMU weniger geeignet. Gründe dafür stellen wahrscheinlich eingeschränkte finanzielle sowie personelle Ressourcen dieser Unternehmen dar, die das Angebot komplexerer digitaler Services erschweren. Zudem geht mit dem Verkauf innovativer digitaler Produkte auch ein großes Investmentrisiko einher, das durch Großunternehmen oder Konzerne besser abgedeckt werden kann. Somit eignet sich für KMU, vor allem bei Markteintritt, vorzugsweise eine Basis- oder Integrations-Plattform mit einer geringeren Anzahl an weniger komplexen Funktionen. Die vorgenommene Analyse hat gezeigt, dass ein Großteil der Unternehmen im deutschen Maschinenund Anlagenbau Plattformen mit geringeren Funktionen und einer geringen Komplexität anbietet. Dies zeigt, dass auch bereits weniger komplexe digitale Plattformen vom Markt angenommen werden und zugleich Wettbewerber für Neueinsteiger darstellen.

Trotz der richtigen Positionierung im Markt ist es besonders wichtig, dass die anbietenden Unternehmen konkrete Ziele für die digitale Plattform formulieren und sich dabei an den Bedürfnissen ihrer Kunden orientieren. Nur so kann sichergestellt werden, dass keine haltlosen Umstrukturierungen des Geschäftsmodells vorgenommen werden, um aufwendige digitale Services anzubieten, die keine Nachfrage finden. Sind die notwendigen Ressourcen im Unternehmen sowie die Nachfrage nach weiteren Plattformfunktionen der Kunden vorhanden, können weitere Funktionen angeboten und die digitale Plattform schrittweise zu einer umfangreichen Plattform ausgebaut werden. Diese Option bietet sich in diesem Fall vor allem dann an, wenn Wettbewerber aus derselben Subbranche des Maschinen- und Anlagenbaus auch umfangreiche Plattformen anbieten.

\subsection{Limitationen}

Bei der dargestellten Analyse muss beachtet werden, dass die verwendeten Daten auf öffentlich zugänglichen Informationen beruhen, die im Zuge einer Internetrecherche bezogen wurden. Dies impliziert, dass sowohl die Eignung der digitalen Plattform für die Analyse als auch das Angebot der einzelnen Funktionen auf der eigenen Einschätzung der Autoren beruht. Zudem liegt bei öffentlichen Informationen eine Beeinträchtigung der Vollständigkeit vor, da einige Daten von den Unternehmen selbst oder aus Pressemitteilungen stammen. Eine weitere Limitation stellt die Anzahl der untersuchten Plattformen dar. Dabei kann davon ausgegangen werden, dass nicht alle digitalen Plattformen im deutschen Maschinen- und Anlagenbau berücksichtigt wurden. 


\subsection{Zukünftige Forschung}

Vor dem Hintergrund der Veränderung des Geschäftsmodells bei der Einführung des Angebots einer digitalen Plattform, deren Umsetzung für viele Unternehmen ein großes Unterfangen darstellt, sollte weiterhin die Entwicklung des Plattformangebots näher untersucht werden. Es kann davon ausgegangen werden, dass einige der untersuchten Unternehmen im Maschinen- und Anlagenbau die digitale Plattform nicht als vollständig neue disruptive Innovation in ihr Angebot aufgenommen haben, sondern dass die Plattform eine Weiterentwicklung bereits bestehender Technologien (z. B. zur Auswertung von Sensordaten) darstellt. Dies würde insbesondere die praktischen Implikationen der Analyseergebnisse ergänzen, indem Unternehmen bei den strategischen Entscheidungen für das Angebot einer digitalen Plattform unterstützt werden. Eine Weiterentwicklung bereits angebotener Technologien hat einen geringeren Einfluss auf das Geschäftsmodell, was insbesondere für kleinere Unternehmen mit geringeren Ressourcen nützlich ist. Das Ergebnis einer solchen weitreichenderen Untersuchung würde bspw. ein Reifegrad- und Entwicklungsmodell digitaler Plattformen im Maschinen- und Anlagenbau darstellen, welches sowohl die verschiedenen Plattformtypen der Clusteranalyse als Abstufungen als auch die Technologien enthält, die bereits in vielen Unternehmen angeboten und weiterentwickelt werden.

\section{Zusammenfassung}

Das Angebot digitaler Plattformen ist mittlerweile auch im Maschinen- und Anlagenbau weit verbreitet. Dabei konnte in den letzten Jahren der Trend verzeichnet werden, dass die Herstellerunternehmen von Maschinen und Anlagen nicht mehr ausschließlich physische Produkte veräußern, sondern zusätzliche auf das Produkt abgestimmte Dienstleistungen, wie bspw. digitale Services. Dieser Wandel kann einen großen Einfluss auf die Veränderung des Geschäftsmodells haben und je nach Komplexität der digitalen Plattformen unterschiedliche Ausmaße annehmen. In diesem Beitrag wurde eine Klassifizierung der digitalen Plattformen im deutschen Maschinen- und Anlagenbau vorgenommen, mithilfe derer unterschiedliche Plattformtypen auf Grundlage ihrer Funktionen identifiziert werden konnten. Diese Plattformtypen weisen verschiedene Funktionskombinationen, und damit verbundene Komplexitätsstufen, auf und können mithilfe von Einflussfaktoren, wie bspw. der Branche der anbietenden Unternehmen, weiter charakterisiert werden. Diese Einteilung unterschiedlicher Plattformtypen kann Unternehmen im Maschinen- und Anlagenbau dabei unterstützen, strategische Entscheidungen bezüglich der Entwicklung und des Angebots digitaler Plattformen zu treffen und eine Einordnung ihrer digitalen Plattform im Wettbewerb vorzunehmen.

Zusatzmaterial online Zusätzliche Informationen sind in der Online-Version dieses Artikels (https://doi. org/10.1365/s40702-021-00810-3) enthalten.

Funding Open Access funding enabled and organized by Projekt DEAL. 
Open Access Dieser Artikel wird unter der Creative Commons Namensnennung 4.0 International Lizenz veröffentlicht, welche die Nutzung, Vervielfältigung, Bearbeitung, Verbreitung und Wiedergabe in jeglichem Medium und Format erlaubt, sofern Sie den/die ursprünglichen Autor(en) und die Quelle ordnungsgemäß nennen, einen Link zur Creative Commons Lizenz beifügen und angeben, ob Änderungen vorgenommen wurden.

Die in diesem Artikel enthaltenen Bilder und sonstiges Drittmaterial unterliegen ebenfalls der genannten Creative Commons Lizenz, sofern sich aus der Abbildungslegende nichts anderes ergibt. Sofern das betreffende Material nicht unter der genannten Creative Commons Lizenz steht und die betreffende Handlung nicht nach gesetzlichen Vorschriften erlaubt ist, ist für die oben aufgeführten Weiterverwendungen des Materials die Einwilligung des jeweiligen Rechteinhabers einzuholen.

Weitere Details zur Lizenz entnehmen Sie bitte der Lizenzinformation auf http://creativecommons.org/ licenses/by/4.0/deed.de.

\section{Literatur}

ads-tec Industrial IT Big-LinX IIoT service \& maintenance plattform. https://www.ads-tec-iit.com/iiotplattform/big-linx.html. Zugegriffen: 31. Okt. 2021

Backhaus K, Erichson B, Plinke W, Weiber R (2018) Multivariate Analysemethoden: Eine anwendungsorientierte Einführung. Springer, Berlin Heidelberg https://doi.org/10.1007/978-3-662-56655-8

Bender B, Lass S, Habib N, Scheel L (2021) Plattform-Bereitstellungsstrategien im Maschinen- und Anlagenbau: Strategien deutscher Unternehmen im Industrie 4.0-Kontext. HMD Praxis der Wirtschaftsinformatik 58:645-660. https://doi.org/10.1365/s40702-020-00648-1

Bollhöfer E, Buschak D, Moll C (2016) Dienstleistungsbasierte Geschäftsmodelle für Industrie 4.0 - aktueller Stand und Potenziale für KMU. Multikonferenz Wirtschaftsinform 3:1287-1298

Demont A, Paulus-Rohmer D (2017) Industrie 4.0-Geschäftsmodelle systematisch entwickeln. In: Digitale Transformation von Geschäftsmodellen. Springer Gabler, Wiesbaden, S 97-125 https://doi.org/10. 1007/978-3-658-12388-8_4

GF Machining Solutions Management SA rConnect-digitale services. https://www.gfms.com/de-de/ services/modernize/digital-services.html. Zugegriffen: 31. Okt. 2021

Grenzebach Digital SERICY Die Digitalisierungs- und Entwicklungs-Plattform für den Mittelstand. Easy. Open. Secure. https://www.grenzebach.digital/de/. Zugegriffen: 31. Okt. 2021

Heidelberger Druckmaschinen AG Heidelberg Assistant. https://www.heidelberg.com/global/de/services_ and_consumables/digital_platforms/heidelberg_assistant/heidelberg_assistant_1.jsp. Zugegriffen: 31. Okt. 2021

Jun C, Lee JY, Yoon J-S, Kim BH (2017) Applications' integration and operation platform to support smart manufacturing by small and medium-sized enterprises. Procedia Manuf 11:1950-1957. https:// doi.org/10.1016/j.promfg.2017.07.341

Kammler F, Remark F, Thomas O (2018) Serviceplattformen als Digitalisierungsstrategie - Entwicklung und Anwendung eines Anforderungskatalogs für den Maschinen-und Anlagenbau. Multikonferenz Wirtschaftsinformatik, Lüneburg, 06.-09. März 2018

KUKA AG KUKA Connect - Roboterdaten, in verwertbare Einblicke umgewandelt. https://connect.kukarobotics.cn/de/. Zugegriffen: 31. Okt. 2021

Lüttenberg H, Beverungen D, Poniatowski M, Kundisch D, Wünderlich NV (2021) Drei Strategien zur Etablierung digitaler Plattformen in der Industrie. Wirtschaftsinformatik Manag 13(2):120-131. https:// doi.org/10.1365/s35764-021-00324-z

Mader GmbH \& Co. KG Machen Sie Ihr Druckluftsystem smarter mit Druckluft 4.0. https://www.mader. eu/loesungen/druckluft-digitalisieren/druckluft-40. Zugegriffen: 31. Okt. 2021

Oberländer AM, Übelhör J, Häckel B (2019) IIoT-basierte Geschäftsmodellinnovation im Industrie-Kontext: Archetypen und praktische Einblicke. HMD 56(6):1113-1125. https://doi.org/10.1365/s40702019-00570-1

Obermaier R, Mosch P (2019) Digitale Plattformen - Klassifizierung, ökonomische Wirkungslogik und Anwendungsfälle in einer Industrie 4.0. In: Handbuch Industrie 4.0 und Digitale Transformation. Springer Gabler, Wiesbaden, S 379-417 https://doi.org/10.1007/978-3-658-24576-4_17

Pflaum A, Schulz E (2018) Auf dem Weg zum digitalen Geschäftsmodell. HMD 55(2):234-251. https:// doi.org/10.1365/s40702-018-0401-2 
Statistisches Bundesamt (2021) Umsatz im deutschen Maschinenbau nach ausgewählten Sektoren in den Jahren 2019 und 2020. In: Statista. https://de.statista.com/statistik/daten/studie/173637/umfrage/ branchenumsatz-des-maschinenbaus-in-deutschland-nach-sektoren/\#professional. Zugegriffen: 20. Mai 2021

Telekom Deutschland GmbH Certuss Dampfautomaten GmbH \& CO. KG - Vernetzung macht der Wartung Dampf. https://geschaeftskunden.telekom.de/vernetzung-digitalisierung/referenzen/ dampfautomaten-certuss. Zugegriffen: 31. Okt. 2021

VDMA, Deutsche Messe AG, \& Roland Berger (2018) Plattformökonomie im Maschinenbau Herausforderungen - Chancen - Handlungsoptionen

Venjakob Maschinenbau Industrie 4.0 im Maschinenbau. https://www.venjakob.de/de/anlagenbau/industrie4-0/. Zugegriffen: 31. Okt. 2021

Weidmann M (2019) Druckluftanlage ans Internet der Dinge: Bitte reparieren. In: Telekom.com. https:// www.telekom.com/de/medien/medieninformationen/detail/druckluftanlage-ans-internet-der-dingebitte-reparieren-581204. Zugegriffen: 31. Okt. 2021 